\title{
Ignition and Extinction Fronts in Counterflowing Premixed Reactive Gases
}

\author{
J. DAOU* and A. LIÑ́́N \\ Dpto. Motopropulsion y Termofluidodinamica, Universidad Politécnica de Madrid, E.T.S.I. Aeronáuticos, Plaza \\ Cardenal Cisneros, 3, 28040 Madrid, Spain
}

\begin{abstract}
We describe two-dimensional steady propagating flame fronts in the stagnation mixing layer between two opposed streams of the same reactive mixture, the propagation taking place in the direction perpendicular to the plane of strain. The front, which is curved by the nonuniform flow field, separates a chemically frozen region from a region with a twin-flame configuration. The front velocity is calculated in terms of the Lewis number, $L e_{F}$, and the Damköhler number, $D a . D a$, equal to the inverse of the Karlovitz number, is defined as the ratio of the strain time to the transit time through the planar unstrained flame. For the cases corresponding to large $D a$, difficult to tackle numerically, analytical expressions are given, characterizing the flame shape, and the variation of the burning rate along the flame front from the nose up to the planar trailing branches. For moderately large and low values of $D a$, the study is carried out numerically, yielding, in particular, the propagation velocity in terms of $D a$, for different values of $L e_{F}$. Different combustion regimes are thus described including flames propagating toward the unburnt mixture, or ignition fronts, standing flames and retreating flames, or extinction fronts. We also describe stationary cylindrical flames of finite-extent, or 2D burning spots. In particular, a critical Lewis number is found, below which negative propagation speeds do not exist while the 2D burning spots mentioned may be encountered. Typically, these exist only for sufficiently small $L e_{F}$ if the $D a$ is within a range $\left[D a_{\min }, D a_{\max }\right]$, depending on $L e_{F}$. For $D a<D a_{\min }$, the $2 \mathrm{D}$ spots are quenched, whereas as $D a$ is increased, they grow in size, tending to give birth to propagating (ignition) fronts; $D a_{\text {max }}$ is indeed found to be the smallest $D a$ allowing for ignition fronts. We notice that the range of existence of the 2D spots, for a given $L e_{F}$, can overlap with that of retreating (extinction) fronts, and possibly with that of $3 \mathrm{D}$ spots, or flame balls, in this flow. However, the 3D case is not addressed in this work.
\end{abstract}

\section{INTRODUCTION}

In most combustion applications, the phenomena of ignition, extinction, and flame propagation are interlinked and occur in highly complex, generally turbulent flows. One important illustrative instance, frequently encountered in practice, is the process of flame initiation by a localized deposit of energy, as for example by a spark in gasoline engines, for which the three phenomena would play an important part in the success of ignition or its failure. Although the analysis of such combustion events is a formidable task in real flow situations, or even in general laminar flows, it is instructive toward their understanding, to examine these events in simple prototypical flows.

As an illustration of this approach, we shall analyze in this paper a type of flame front

\footnotetext{
${ }^{*}$ Corresponding author. Present address: Engineering Science and Applied Mathematics Department, Northwestern University, 2145 Sheridan Road, Evanston, IL 60208-3125, USA. E-mail: daou@nwu.edu
}

structure that may be encountered when in a two-dimensional flow configuration consisting of the same reactive mixture as sketched in Fig. $1 \mathrm{~A}$, ignition is brought about by a hot spot near the stagnation line. If in this flow configuration the velocity components are given by $v_{Y}=-a Y$ and $v_{Z}=a Z$ in the absence of chemical reaction, where $a$ is the strain rate, the generated flame will propagate rapidly in the $Z$ direction, aided by the flow, while its propagation in the $Y$-direction will be slowed by the opposing flow, thus leading to standing planar fronts. However flame propagation in the negative and positive $X$-direction will be neither aided nor opposed by the flow. As a consequence, the flame will extend along the $X$-axis in the form of two propagating fronts, curved in the $Y$-direction, which will be soon nearly aligned with the $Z$-axis and independent of the $Z$-coordinate, for finite $Z$.

The present analysis will address the propagation of such fronts along the $X$-direction, for an Arrhenius reaction and using, for simplicity, the thermodiffusive approximation of constant 


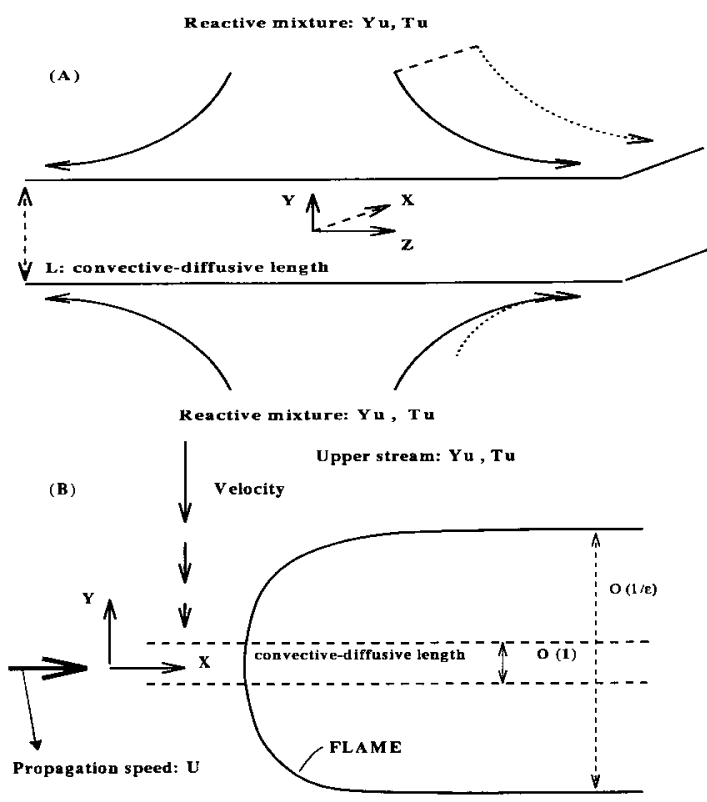

Fig. 1. (A) Opposed streams of the same reactive mixture, with temperature $T_{u}$ and a fuel mass fraction $Y_{u}$. The flow is two-dimensional, with velocity components $v_{Y}=-a Y$ and $v_{Z}=a Z$ on the $Y$-axis and $Z$-axis, respectively. (B) A two-dimensional front (independent of $Z$ ), propagating along the $X$ direction. The trailing planar branches correspond to the strongly burning twin-flame solution (independent of $X$ and $Z$ ). The horizontal arrow represents the $X$-component of velocity in a frame attached to the flame front, and $\epsilon$ is the typical thickness of the flame, relative to the convective-diffusive length.

density. If the cold boundary difficulty is remedied, say by the introduction of an ignition temperature, then similarity solutions for the temperature and composition fields are known to exist in the present flow configuration, dependent only on $Y$. Two such stable solutions, corresponding respectively to a frozen and a strongly burning mode, are generally found, if the activation energy is large enough, and the strain rate below an extinction value. In this paper, we shall describe the steady flame front that propagates in the negative $X$-direction with traveling wave solutions independent of the $Z$-coordinate which converts one of these modes into the other. If the frame of reference is attached to the flame front, then the solutions are also independent of time, and the velocity field is given by $\vec{v}=(U,-a Y, a Z)$, where the additional component, $U$, is an eigenvalue representing the propagation speed. The flames under consideration will correspond to ignition fronts extending combustion to near-frozen regions, if $U>0$, and to extinction fronts otherwise. The effects of density changes due to gas expansion on the front velocity and its structure are not addressed in this paper, neither is the stability of the flame propagation process.

In addition to the study of these fronts, we shall discuss, also within the framework of the thermodiffusion model, another combustion mode corresponding to two-dimensional finiteextent burning solutions, in conditions where the strain rate is close to or higher than the extinction value of the planar twin flame.

The paper is organized as follows. The next section is dedicated to a formulation of the problem, followed by presentation of the results, in terms of a Damköhler number, $D a$, and a Lewis number. An analytical description is given first, for the cases corresponding to large values of $D a$, difficult numerically, completed by a numerical description covering a wide range of $D a$ and different values of the Lewis number.

\section{MODEL AND FORMULATION}

The analysis examines the propagation of twodimensional combustion fronts, between two opposed streams of the same reactive mixture, along the direction $X$ perpendicular to the plane of strain, as sketched in Fig. 1. For simplicity, the density and transport properties are assumed constant, and the combustion is represented by an irreversible reaction of the form

$F \rightarrow P+q$,

where $F$ denotes the fuel, $P$ the products, and $q$ the heat released per unit mass of fuel. The combustion rate, $\omega$, defined as the mass of fuel consumed by unit volume and unit time is assumed to follow an Arrhenius law of the form $\omega=B \rho Y_{\Gamma} e^{-l i / R T}$,

where $B, \rho, Y_{F}, T, E$, and $R$ represent respectively the preexponential factor, the density, the mass fraction of fuel, the temperature, the activation energy, and the universal gas constant.

As already noted, for the existence of the 
steadily propagating flame fronts under consideration, the activation energy of the reaction must be large enough so that two steady stable combustion modes independent of $X$ are obtained for strain rates below an extinction value. The front joins these two modes, corresponding to a frozen and a strongly burning solution of the planar twin flame, respectively.

The conservation equations are

$$
\begin{aligned}
& U \frac{\partial Y_{F}}{\partial X}=D_{F} \Delta Y_{F}-\frac{\omega}{\rho}+a Y \frac{\partial Y_{F}}{\partial Y} \\
& U \frac{\partial T}{\partial X}=D_{T} \Delta T+\frac{q}{c_{p}} \frac{\omega}{\rho}+a Y \frac{\partial T}{\partial Y},
\end{aligned}
$$

where $D_{F}$ and $D_{T}$ denote the diffusion coefficients of the fuel and of heat, and $c_{p}$ the heat capacity.

For a nondimensional description, we shall use the normalized quantities

$y_{F}=\frac{Y_{F}}{Y_{u}}$ and $\theta=\frac{T-T_{u}}{q Y_{u} / c_{p}}$,

and select as reference velocity the laminar burning speed of a planar flame, ${ }^{1} S_{L}^{0}$, and as unit length the convection-diffusion length $L=$ $\sqrt{2 D_{T} / a}$. Hence, the governing equations take the form

$$
\begin{aligned}
& U \frac{\partial y_{F}}{\partial x}=\frac{\epsilon}{L e_{F}} \Delta y_{F}-\epsilon^{-1} \tilde{\omega}+2 \epsilon y \frac{\partial y_{F}}{\partial y} \\
& U \frac{\partial \theta}{\partial x}=\epsilon \Delta \theta+\epsilon^{-1} \tilde{\omega}+2 \epsilon y \frac{\partial \theta}{\partial y},
\end{aligned}
$$

where $L e_{F}=D_{T} / D_{F}$ is the Lewis number, and $\epsilon$ represents the ratio of the premixed flame thickness, $l_{F l}^{0}=D_{T} / S_{L}^{0}$, to the reference length $L$, and can be used to define a relevant ${ }^{2}$ Damköhler number, $D a$, by

$\epsilon \equiv \frac{l_{F l}^{0}}{L}=\frac{l_{F l}^{0}}{\sqrt{2 D_{T / a}}}, D a \equiv \epsilon^{-2}$

The boundary conditions adopted are
$y_{F}-1=\theta=0$ for $x \rightarrow-\infty$ or $|y| \rightarrow \infty$,

corresponding to the frozen solution, and

$\frac{\partial y_{F}}{\partial x}=\frac{\partial \theta}{\partial x}=0$ for $x \rightarrow \infty$,

since, downstream, the profiles tend to the strongly burning solution of the planar twin flame, independent of $x$.

The nondimensional reaction rate, $\tilde{\omega}$, is given by

$\tilde{\omega}=\frac{\beta^{2}}{2} y_{F} \exp \left(\frac{\beta(\theta-1)}{1+\alpha(\theta-1)}\right)$,

where $\alpha \equiv\left(T_{a d}-T_{u}\right) / T_{a d}$ is a heat-release parameter, and $\beta=E\left(T_{a d}-T_{u}\right) / R T_{a d}^{2}$ the Zeldovich number; here $T_{a d}=T_{u}+q Y_{u} / c_{p}$ is the adiabatic flame temperature. The main object of the analysis is the determination of the propagation velocity $U$ of the flame fronts, along with the range of existence of the finiteextent, two-dimensional steady burning spots mentioned in the introduction. The results will be presented in terms of $\epsilon=D a^{-1 / 2}$ and $l_{F}=$ $\beta\left(L e_{F}-1\right)$. In the numerical study, the parameters $\beta$ and $\alpha$ will have fixed values, namely $\beta=$ 8 and $\alpha=0.85$.

\section{SOLUTION FOR LARGE DAMKÖHLER NUMBERS}

In this section, we briefly discuss the large Damköhler number cases, which are difficult to obtain numerically, since the flame is then thin and extends over a large domain, ${ }^{3} y \sim \epsilon^{-1}$. In the limit $\epsilon \rightarrow 0$, the flame can be viewed as an infinitely thin surface with typical width of order $\epsilon$ on the scale $L$, and with a normal velocity relative to the fresh mixture $S(y)$. For those cases, an analytical description can be obtained, yielding in particular the propagation eigenvalue, $U$, the normal flame speed, $S(y)$, and the local flame temperature, $\theta_{F l}$, by the expressions

${ }^{3}$ For $\epsilon \rightarrow 0$, the $y$-locations of the trailing branches of the flame, corresponding to the planar twin-flame, are determined by the requirement that the $y$-component of velocity is equal to $S_{L}^{0}$ there, which leads, in nondimensional form, to $y \sim \epsilon^{-1}$. 


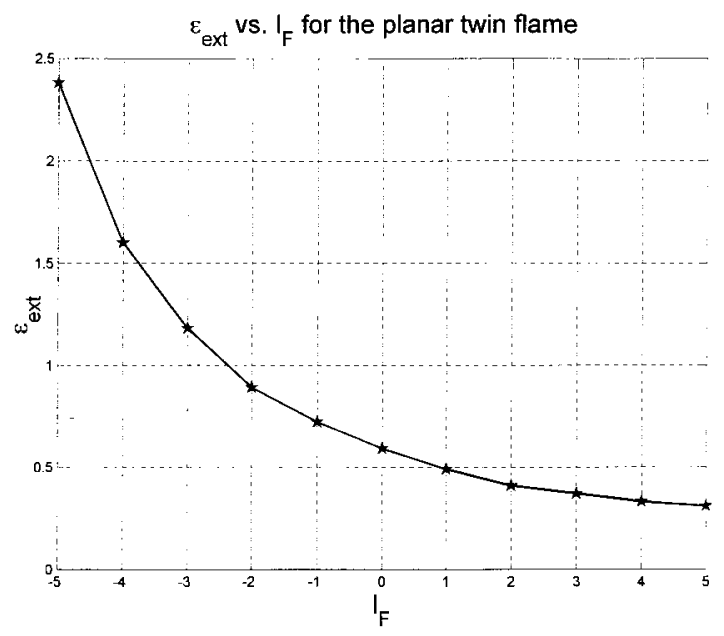

Fig. 2. The value of $\epsilon, \epsilon_{\text {ext }}$, corresponding to the extinction of the planar twin flame, versus $l_{F}$. The values of the other parameters, kept fixed in the present study, are $\beta=8$ and $\alpha$ $=0.85$.

$U=1-4\left(1+\frac{l_{F}}{2}\right) \epsilon^{2}$

$S(y)=1-\left(1+\frac{l_{F}}{2}\right) \frac{4 \epsilon^{2}}{1+(2 \epsilon y)^{2}}$

$\theta_{F l}=1-\frac{l_{F}}{\beta} \frac{4 \epsilon^{2}}{1+(2 \epsilon y)^{2}}$

A simple way to justify these expressions is to use already established results on stretched flames, instead of repeating lengthy derivations. To this end, we first introduce some notations. Let $\tilde{x}=\epsilon x$ and $\tilde{y}=\epsilon y$ be rescaled variables and let the flame surface be described by $\tilde{x}=f(\tilde{y})$ so that its unit normal vector pointing to the burnt gas is defined by

$\vec{n}=\frac{\vec{\imath}-f^{\prime}(\tilde{y}) \vec{j}}{\sqrt{1+f^{\prime}(\tilde{y})^{2}}}$,

and the local burning speed, $S$, is given by

$S=(U \vec{i}-2 \tilde{y} \vec{j}) \cdot \vec{n}=\frac{U+2 \tilde{y} f^{\prime}(\tilde{y})}{\left(1+f^{\prime}(\tilde{y})^{2}\right)^{1 / 2}}$

Anticipating that the deviation of the normal flame speed from 1 (the unstretched planar value) is small, for $D a \gg 1$, a first approximation for $U$ and $f^{\prime}$, say $U_{0}$ and $f_{0}^{\prime}$, can be obtained upon replacing $S$ by 1 in the preceding equation,
$1=\frac{U_{0}+2 \tilde{y} f_{0}^{\prime}(\tilde{y})}{\left(1+f_{0}^{\prime}(\tilde{y})^{2}\right)^{1 / 2}}$

Since the freely propagating flame is expected to be smooth, and symmetrical relative to the $x$-axis, it follows that $f_{0}^{\prime}(0)=0$, which, on using Eq. 9, leads to ${ }^{4}$

$U_{0}=1$ and $f_{0}^{\prime}(\tilde{y})=\frac{4 \tilde{y}}{1-4 \tilde{y}^{2}}$,

showing, in particular, that the trailing branches of the flame are located at $|\tilde{y}|=|\epsilon y|=\frac{1}{2}$. For an improved description, we shall use, with our notations, the following established results [1-3],

$S=1-\left(1+\frac{l_{F}}{2}\right) \kappa$ and $\theta_{F l}=1-\frac{l_{F}}{\beta} \kappa$,

valid for small values of $\kappa$, the flame stretch nondimensionalized by the flame time $l_{F l}^{0} / S_{I}^{0}$, given by [4]

$\kappa=-\vec{n} \cdot \nabla \times(\vec{v} \times \vec{n})+\left(\vec{v}_{\text {flame }} \cdot \vec{n}\right)(\nabla \cdot \vec{n})$

Here $\vec{v}_{\text {flame }}$ denotes the velocity of the flame and $\vec{v}$ the velocity of the fluid immediately ahead of it, which in our frame of reference are given by $\vec{v}_{\text {llame }}=\overrightarrow{0}$ and $\vec{v}=U \vec{\imath}-2 \tilde{y} \vec{j}+2 \tilde{z} \vec{k} ; \nabla$ $=\epsilon^{2}(\partial / \partial \tilde{x}, \partial / \partial \tilde{y}, \partial / \partial \tilde{z})$ is the gradient operator nondimensionalized by $l_{F}^{0}$. When these expressions are substituted into Eq. 12, and use is made of Eq. 10 to approximate $U$ and $f^{\prime}$, the following expression for $\kappa$ is found:

$\kappa=\left[\frac{f_{0}^{\prime \prime}}{\left(1+f_{0}^{\prime 2}\right)^{3 / 2}}+\frac{2 f_{0}^{\prime 2}}{1+f_{0}^{\prime 2}}\right] \epsilon^{2}$,

or

$\kappa=\frac{4 \epsilon^{2}}{1+4 \tilde{y}^{2}}=\frac{4 \epsilon^{2}}{1+4 \epsilon^{2} y^{2}}$

Note that the first term in Eq. 13 is the flame curvature which, as it should, tends to zero as the locations of the trailing planar wings are approached, $\tilde{y} \rightarrow \pm \frac{1}{2}$; the second term tends to zero at the leading edge, $\tilde{y}=0$, and to 2 as $\tilde{y} \rightarrow$ $\pm \frac{1}{2}$, where the only contribution to flame stretch

${ }^{4}$ Note that $f_{0}^{\prime}=0$, corresponding to a planar flame propagating in the $x$-direction is a kinematically possible solution, which is rejected for being unstable in the present flow. 

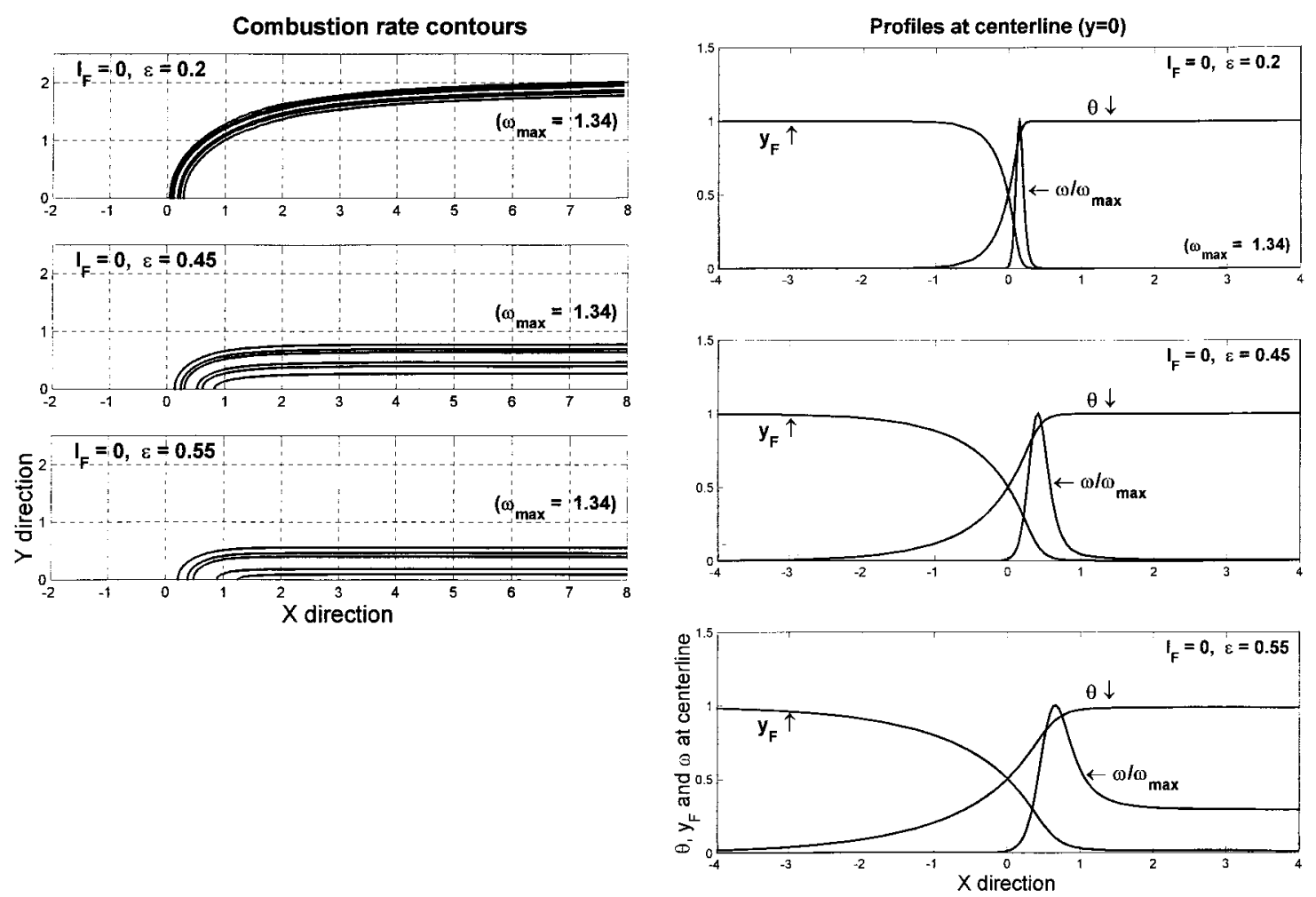

Fig. 3. (a) Comparison for $l_{F}=0$ between three cases corresponding from top to bottom to $\epsilon=0.2,0.45$, and 0.55 , respectively. Plotted in each subfigure are three isocontours of the combustion rate, $\omega$, corresponding to $\omega=0.7 \omega_{\text {max }}, \omega=$ $0.4 \omega_{\max }$, and $\omega=0.1 \omega_{\max }$, where $\omega_{\max }$ is the maximum of $\omega$ (also indicated). As $\epsilon$ is increased, the front evolves from an ignition front to an extinction front (retreating front), with propagation velocities (which can be extracted from Fig. 6) equal to $0.80,-0.08$, and -1.06 , respectively. Of course, the plots extend to negative $y$ by symmetry with respect to $y=0$. (b) Temperature, mass fraction, and combustion rate profiles at centerline, $y=0$, vs. the longitudinal coordinate $x$. The subfigures correspond frame per frame to those of Fig. 3a. Note that the combustion rate has been normalized by its maximum value over the whole domain, $\omega_{\max }$

is due to strain. Finally, when Eq. 14 is used in Eqs. 11, the expressions advanced in Eqs. 7 are obtained, with their significance being clear.

\section{NUMERICAL STUDY}

In this section a synthesis of the numerical results is given, which mainly describe flame propagation along the $x$-direction, and correspond to the governing equations (2) and the boundary conditions (4) and (5). The solutions are obtained iteratively by solving with a multigrid method [5], the steady form of the equations discretized using a finite-volume approach. The flame front velocity $U$ is updated so that the temperature keeps a prescribed value at the origin, which fixes the flame front at $x=0$. The computational domain extent in the trans- verse $y$-direction is typically 10 times the convective-diffusive length, $L=\sqrt{2 D_{T} / a}$; its extent in the $x$-direction is typically 100 times the planar laminar flame thickness, $l_{F l}^{0}$. A nonuniform rectangular grid with typically 100 thousand points is used.

We shall begin by describing, for fixed values of $l_{F}$, the dependence of combustion fronts on $\epsilon$ $=D a^{-1 / 2}$. Then, the essential results will be summarized in a plot of the propagation eigenvalue $U$ vs. $\epsilon$ for different values of $l_{F}$, followed by a discussion of the regimes observed. We shall see that these include propagating, still, and retreating fronts, but also nonpropagating finite-extent, two-dimensional steady structures, for a certain range of the parameters.

As a preliminary step, useful in the discussion below, we have plotted in Fig. 2 the extinction 
Combustion rate contours

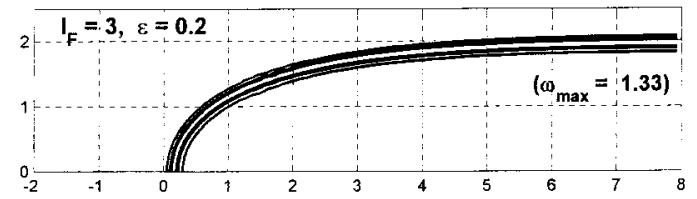

$I_{F}=3, \varepsilon=0.28$
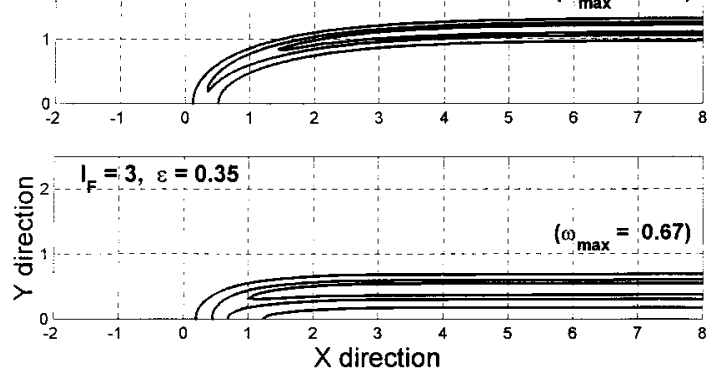

Profiles at centerline $(y=0)$
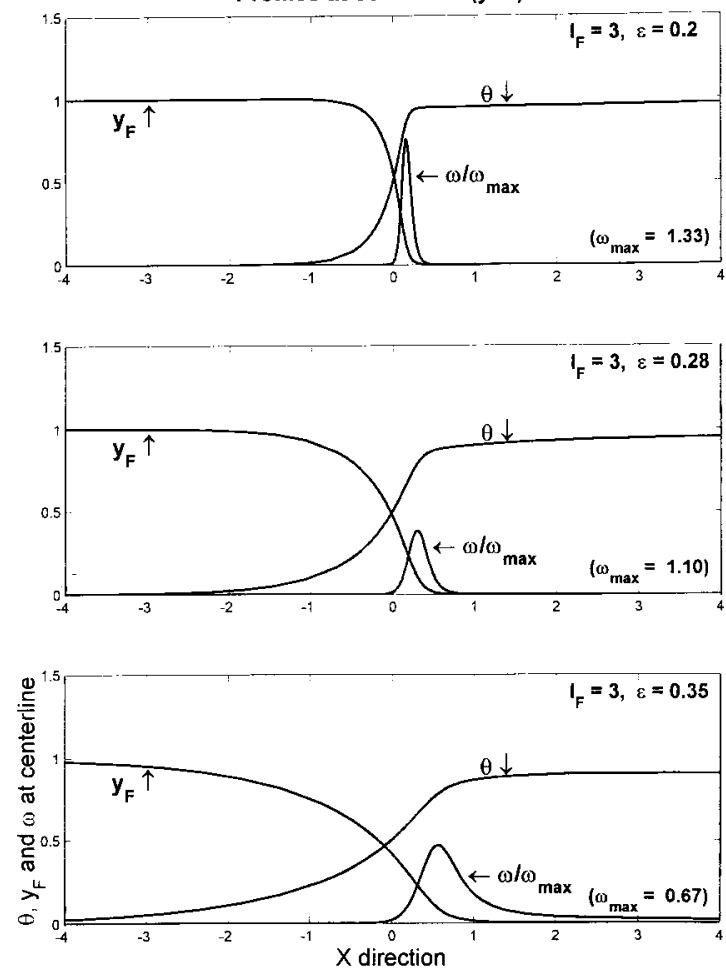

Fig. 4. (a) Comparison for $l_{F}=3$ between three cases corresponding from top to bottom to $\epsilon=0.2,0.28$, and 0.35 , respectively. Plotted in each subfigure are three isocontours of the combustion rate, $\omega$, characterized as in Fig. $3 \mathrm{a}$. The propagation velocities from top to bottom are equal to $0.60,0.07$, and -0.7 , respectively. (b) Temperature, mass fraction, and combustion rate profiles at centerline, $y=0$, vs. the longitudinal coordinate $x$. The subfigures correspond frame per frame to those of Fig. 4 a.

value of the planar twin-flame, $\epsilon_{e x t}$, against $l_{F .}$. This plot is obtained numerically by solving the underlying one-dimensional problem $x$-independent. ${ }^{5}$

\section{Dependence on the Damköhler Number}

In this section we study the dependence on $\epsilon$, for three fixed values of $l_{F}$. We begin with the case $l_{F}=0$, for which three subfigures corresponding from top to bottom to $\epsilon=0.2, \epsilon=$

\footnotetext{
${ }^{5}$ Results describing the (one-dimensional) twin flame can be found e.g. in [6], [3] (page 41), and [10] (page 332). In particular, as stressed in the numerical study in [6], and suggested by the analytical treatment in [3] or [10], extinction typically occurs by quenching (incomplete reaction) at the symmetry surface, $y=0$, except for $L e_{F}$ sufficiently above 1 . In this latter case, the flame extinguishes while the reaction zones are away from the symmetry surface and reaction is complete. More precisely, in our notations and in the limit $\beta \rightarrow \infty$, these two modes of extinction occur for $l_{F}$ $<4$ and $l_{F}>4$, respectively, with $\epsilon_{e x t}$ given by $\epsilon_{e x t}=\pi^{1 / 2}$ $\exp \left(-I_{F} / 4\right) / 2$, for $l_{F}<4$.
}

0.45 , and $\epsilon=0.55$, are shown in Fig. 3a. The values of $\epsilon$ are chosen such that the propagation velocity is that of a moderately curved front (positive), close to zero, or significantly negative (corresponding to $\epsilon \approx \epsilon_{\text {ext }}$ ), from top to bottom, respectively. Each subfigure represents isocontours of the combustion rate, $\omega$, as described in the captions where the propagation velocities are also given. The temperature, mass fraction, and combustion rate profiles at the centerline, $y=0$, corresponding frame by frame to Fig. 3a, are plotted vs. the longitudinal coordinate $x$, in Fig. $3 b$. Note that as $\epsilon$ is increased, the reaction persists behind the leading front, resembling thus the situation behind the edge of a triple flame.

The influence of the Lewis number can be appreciated by comparing the case just presented, corresponding to $l_{F}=0$, with two cases represented in Figs. 4 and Figs. 5 and corresponding to $l_{F}=3$ and $l_{F}=-3$, respectively. 
Combustion rate contours
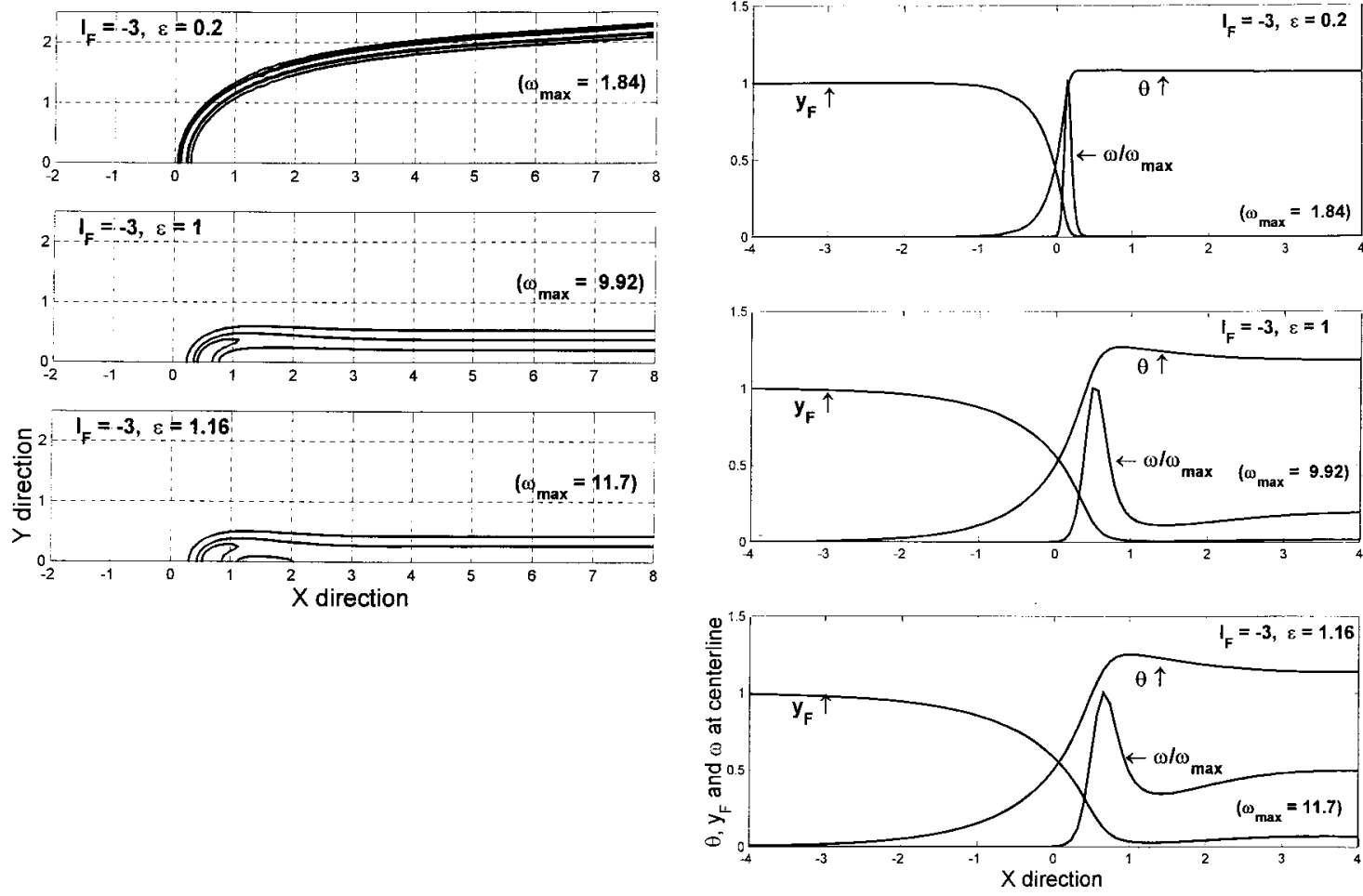

Fig. 5. (a) Comparison for $l_{F}=-3$ between three cases corresponding from top to bottom to $\epsilon=0.2$, 1 , and 1.16 , respectively. Plotted in each subfigure are three isocontours of the combustion rate, $\omega$, characterized as in Fig. $3 \mathrm{a}$. The propagation velocities from top to bottom are equal to $1.19,0.02$, and -1.15 , respectively. (b) Temperature, mass fraction, and combustion rate profiles at centerline, $y=0$, vs. the longitudinal coordinate $x$. The subfigures correspond frame per frame to those of Fig. 5a.

The choice of the values of $\epsilon$, and the notations, explicated in the captions, are similar to those introduced above. For $l_{F}>0$ the minimum flame temperature occurs at the leading edge, where curvature is highest, in agreement with the asymptotic results for small $\epsilon$. This explains the positive slope of the temperature profile behind the edge observed in Fig. 4b. The occurrence of the maximum flame temperature at the leading front, for $l_{F}<0$, and the negative slope of the temperature profile behind the edge in Fig. 5b, are understood in the same fashion.

It is interesting to note that negative propagation speeds have been obtained for the three values of the Lewis number considered. Nevertheless, this is not always so, as we shall now see.

\section{Combustion Regimes}

Shown in Fig. 6 is a plot of the propagation eigenvalue $U$ vs. $\epsilon$ for five values of $l_{F}$, namely,
$l_{F}=-5,-4,-3,0$, and 3 . For each value, $U$ has been normalized by the corresponding planar unstretched flame speed, obtained numerically. The calculated speeds are represented by small circles and have been restricted to $\epsilon \geq 0.2$ to ensure sufficient numerical resolution. Also, for $\epsilon<0.2$, solid curves starting from the point ( $\epsilon=0, U=1)$ and corresponding to the asymptotic expression (7.a) are plotted for the five values of $l_{F}$ given above. ${ }^{6}$

As a first observation relative to the numeri-

'Although the values of $\epsilon$ considered in the numerics are not very small compared to 1 , it is seen that the agreement between the tendencies of the numerical and asymptotic curves is good for $l_{F}=3$ and $l_{F}=0$, and much less satisfactory for $l_{F}=-4$ and -5 . In these latter cases, the bad disagreement is due, at least partly, to the significant overshoot of the flame temperature above 1 , which limits the validity of the linearized expressions (Eqs. 7). A more fundamental issue when the Lewis number is sufficiently small is the expected cellular instability of the front as it 


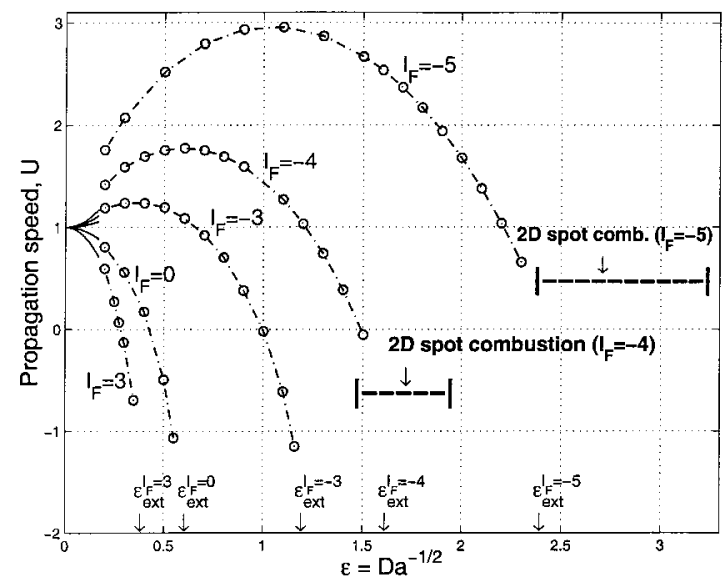

Fig. 6. Propagation speed $U$ versus $\epsilon$ for five values of $l_{F}, l_{F}$ $=-5,-4,-3,0$, and 3 . For each $l_{F}, U$ has been normalized by the corresponding planar (unstretched) flame speed obtained numerically. The calculated speeds are represented by small circles and have been restricted to $\epsilon \geq$ 0.2 to ensure sufficient numerical resolution. For $\epsilon<0.2$, solid curves starting from the point ( $\epsilon=0, U=1)$ and corresponding to the asymptotic expression (7.a) are plotted for the five values of $l_{F}$ given above (the upper curve is for $l_{F}=-5$ and the lower one for $l_{F}=3$ ). The values of $\epsilon, \epsilon_{e x x}$, corresponding to the extinction of the planar structure (given in Fig. 2), are indicated by downward arrows on the $\epsilon$-axis. Two horizontal segments indicate the $\epsilon$-range of existence of steady nonpropagating combustion in the form of two-dimensional burning spots, for $l_{F}=-4$ and $l_{F}=-5$ respectively. These burning spots are described in Fig. 7.

cal results, we note the change in the behavior of $U$ vs. $\epsilon$, as $l_{F}$ is varied. The curves decrease monotonically with $\epsilon$, except when $l_{F}$ is sufficiently negative. For those cases, the value of $U$ initially increases, in agreement with Eqs. 7, leading to an important overshoot above 1, which is attributed to the increase of the burning temperature and intensification of the reaction at the leading front. The physical mechanisms leading to this intensification are well known from the studies on curved and stretched premixed flames. Despite this intensification, the propagation speed ultimately decreases, for sufficiently high values of $\epsilon$, due to the heat losses transverse to the direction of propagation.

Another important observation which can be drawn from Fig. 6 is the existence of a critical negative value of $l_{F}$, say $l_{F}^{*}=\beta\left(L e^{*}-1\right)$,

tends to a locally planar unstretched premixed flame in the limit $\epsilon \rightarrow 0$. This point is not pursued in the present paper. below which negative propagation speeds, or extinction fronts, are impossible. For our choice of the fixed parameters (given in the captions of Fig. 2, for example), this value is seen to lie between -5 and -4 .

Common to each case with $l_{F}>l_{F}^{*}$ is the existence of a range of $\epsilon$ with negative speeds of the front as found for triple flames under strain [7-9]. The upper limit of this range is $\epsilon_{\text {ext }}$, the extinction value of the planar structure, indicated by a downwards arrow on the $\epsilon$-axis. ${ }^{7}$

For $l_{F}<l_{F}^{*}$, say $l_{F}=-5$, the propagation velocity is strictly positive as $\epsilon \rightarrow \epsilon_{e x t}^{-}$. When $\epsilon$ exceeds $\epsilon_{\text {ext }}$, the infinite structure disintegrates since the planar (twin-flame) burning solution far behind the leading edge, $x \gg 1$, does not exist for $\epsilon>\epsilon_{c x t}$. However, this does not necessarily lead to total extinction, since combustion can still persist if $L e_{F}$ is small, as the numerical results indicate, in the form of finiteextent structures, or two-dimensional burning spots. These are similar to the flame balls encountered outside the normal flammability limits, $[10,11]$. To describe these structures, we shall examine the simplest spatial distribution, characterized by a single burning spot in the flow. For $l_{F}=-5$, such an isolated spot ${ }^{8}$ exists if $\epsilon$ is, roughly, in the range [2.4-3.2], represented by a horizontal line segment in Fig. 6 . This range is reduced for $l_{F}=-4$, as indicated in the same figure. For smaller values of $l_{F}$, say $l_{F}<-3$, such modes of combustion are not found, according to numerical experimentation.

For illustration, Fig. 7 depicts, for three cases corresponding to $l_{F}=-5$ and to different values of $\epsilon$, the burning spots under discussion. In Figs. $7 \mathrm{a}$ and $7 \mathrm{~b}$ combustion rate and temperature contours are plotted, respectively, which should be completed by symmetry about the $x$ and $y$-axes. The corresponding mass fraction, temperature, and combustion rate profiles at the centerline, $y-0$, are given in Fig. 7c. As $\epsilon$ is increased beyond the value of the bottom subfigure, total extinction of the two-dimensional structure results; as $\epsilon$ is decreased below its value in the upper subfigure, the numerical

\footnotetext{
${ }^{7} \epsilon_{e x t}$ is extracted from Fig. 2.

${ }^{8}$ The burning spot solution corresponds to the governing equations (2) with $U=0$ and the far-field boundary condition $\theta=1-y_{F}=0$.
} 


\section{Combustion rate contours}
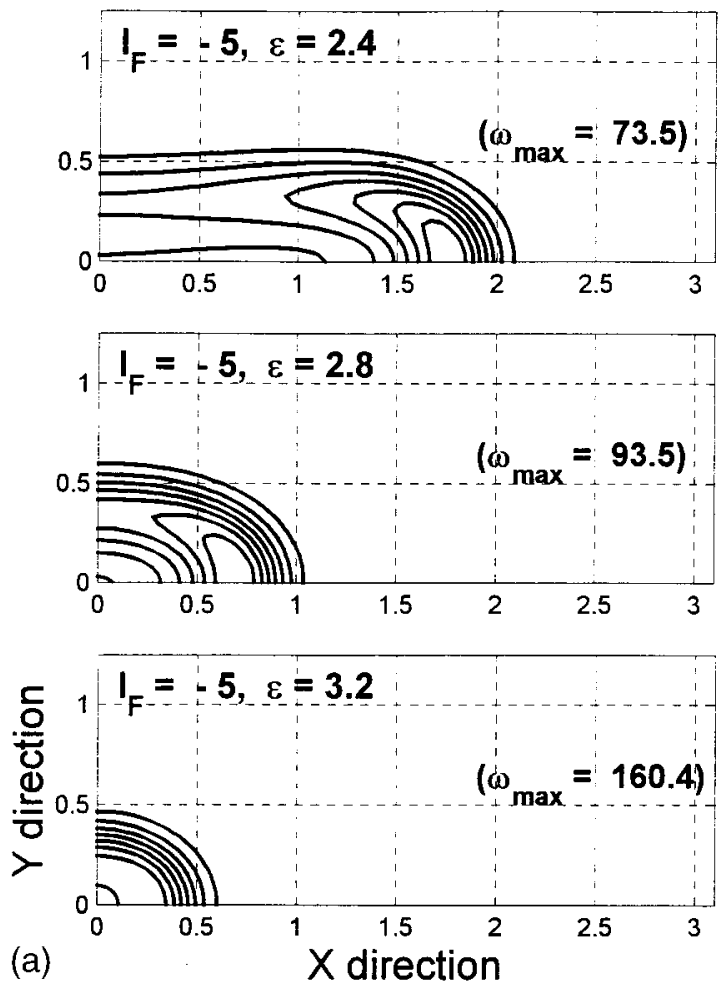

(a)
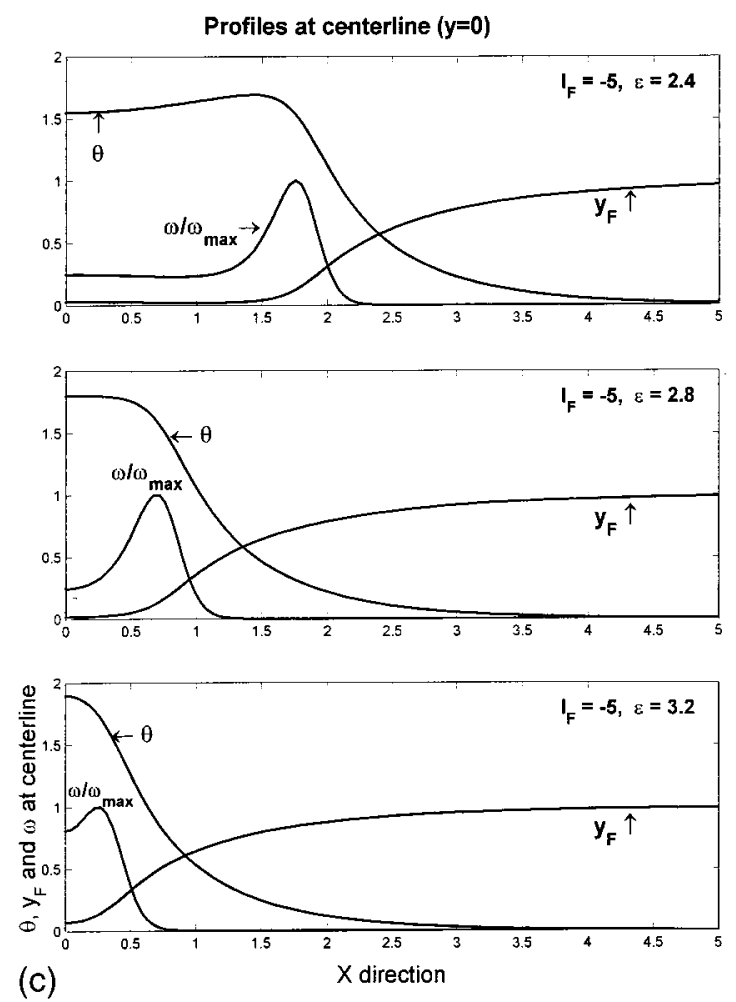

\section{Temperature contours}
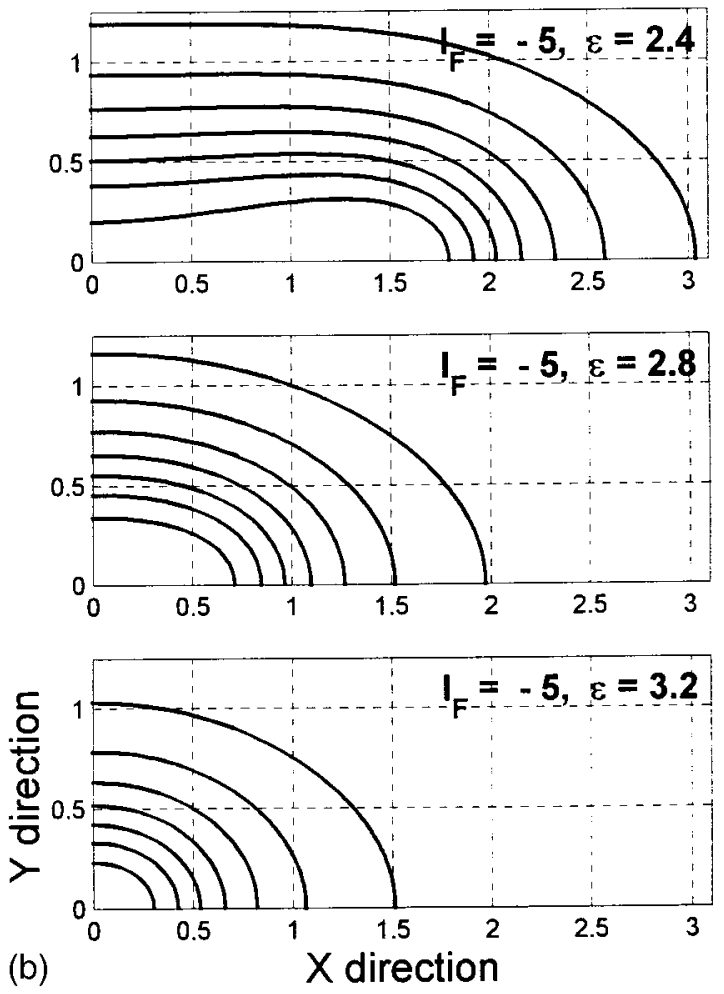

Fig. 7. (a) Combustion rate contours associated with $2 \mathrm{D}$ burning nonpropagating spots corresponding to $l_{F}=-5$ and, from top to bottom, to $\epsilon=2.4,2.8$, and 3.2, respectively. In each subfigure, which are to be completed by symmetry about the $x$-axis and $y$-axis, six isocontours of the combustion rate, $\omega$, are plotted equidistributed between zero and the maximum of $\omega, \omega_{\max }$ (also indicated). As $\epsilon$ is increased beyond the value of the bottom subfigure, total extinction of the two-dimensional structure results; as $\epsilon$ is decreased below its value in the upper subfigure, the infinite extent burning structure is recovered. (b) Same as Fig. 7a, with temperature contours being plotted instead of combustion rate contours. (c) Temperature, mass fraction, and combustion rate profiles at centerline, $y=0$, vs. the longitudinal coordinate $x$. The subfigures correspond frame per frame to those of Figs. $7 \mathrm{a}$ and $7 \mathrm{~b}$. 
results suggest the recovery of the infiniteextent burning structure. Note that, for $l_{F}=$ -5 , the ranges of existence of the finite-extent spots and infinite-extent fronts do not overlap. Nevertheless these ranges do overlap for $l_{F}=$ -4 (Fig. 6). In this latter case, the 2D spot combustion regime extends down to the value of $\epsilon$ corresponding to the zero-propagation velocity of the flame fronts, described above.

A remark is here in order. We have just seen, when the ignition-extinction fronts disintegrate, that combustion is still able to persist in the form of $2 \mathrm{D}$ spots, in a certain domain of the $(\epsilon$, $\left.l_{F}\right)$ plane. We have also observed that an overlap of the domains of existence of the $2 \mathrm{D}$ spots, and propagating fronts regimes is possible. In the same fashion, one may expect that combustion in the form of 3D spots, or deformed flame balls, could be the only possible regime in certain circumstances, with a possible overlap with the aforementioned regimes. Although this point is not investigated in the present study, it is relevant to note that such flame balls stabilized by the two-dimensional flow considered herein, or more generally by flows that are linear functions of the coordinates, have been described by Buckmaster and Joulin [12]. Finally, it is instructive to examine the similarities and complementary aspects between the present results and the closely related recent findings in [13-15].

\section{CONCLUDING REMARKS}

In this work, we have described the possibility of flame propagation in a two-dimensional counterflow of premixed reactive gases, in a direction perpendicular to the plane of strain. The findings can be used to analyze with some detail ignition and extinction events in the idealized flow under consideration, a step toward a better understanding of such events in realistic, more complicated flows. The results have shown the existence of ignition and extinction fronts in premixed systems which share many similarities with their counterparts, the triple flames, in unpremixed systems. Those similarities are particularly apparent, for example, if one plots the propagation velocity $U$ against a suitably de- fined Damköhler number, and for different values of the Fuel Lewis number. Without underlining here the main findings, given in the abstract, we shall simply illustrate their use in an "ignition" problem. Consider the evolution of combustion, in the flow under consideration, from an initial two-dimensional hot spot of burnt gases. Then Fig. 6, representing $U$ vs. $\epsilon=$ $D a^{-1 / 2}$, suggests the following conclusions. For ignition to be successful, it is necessary in usual mixtures, characterized by a Lewis number close to 1 , that $\epsilon$ be such that the corresponding $U$ in Fig. 6 be positive. Successful ignition in this case leads to propagating fronts. However, if the Lewis number is sufficiently small, successful ignition may result in steadily burning finiteextent, two-dimensional spots, if $\epsilon$ is within a given range.

\section{REFERENCES}

1. Clavin, P., Prog. Energy Combust. Sci. 11:1-59 (1985).

2. Matalon, M., and Matkowsky, B. J., JFM 124:239-259 (1982).

3. Buckmaster, J., and Ludford, G. S., Lectures on Mathematical Combustion, SIAM Press, Philadelphia, 1983, p. 41.

4. Matalon, M., Combust. Sci. Technol. 31:169 (1983).

5. Ruge, J., and Stüben, K., in Proc. of Multigrid Conf., Bristol, Sept. 1983.

6. Sato, J., and Tsuji, H., Combust. Sci. Technol. 33:193 (1983).

7. Hartley, L. J., and Dold, J. W., Combust. Sci. Technol. 80:23 (1990).

8. Kioni, P. N., Rogg, B., Bray, C., and Liñán, A., Combust. Flame 95:276-290 (1993).

9. Daou, J., and Liñán, A., Combust. Theory Modelling, 2, 449-477 (1998).

10. Zeldovich, Y. B., Barenblatt, G. I., Librovich, V. B., and Makhviladze, G. M., The Mathematical Theory of Combustion and Explosions, Consultants Bureau, New York, 1985, p. 332.

11. Ronney, P. D., Combust. Flame 82:1-14 (1993).

12. Buckmaster, J., and Joulin, G., JFM 227:407-427 (1991).

13. Liu, J. B., and Ronney, P. D., Premixed edge-flames in spatially-varying straining flows Combust. Flame (in press).

14. Vedarajan, T. G. and Buckmaster, J. D., Combust. Flame 114:267 (1998).

15. Shay, M. L. and Ronney P. D. Combust. Flame 112: 171 (1998).

Received 21 July 1998; revised 18 November 1998; accepted 9 December 1998 